\title{
Expression of Angiostatin cDNA in a Murine Fibrosarcoma Suppresses Primary Tumor Growth and Produces Long-Term Dormancy of Metastases
}

\author{
Yihai Cao, ${ }^{\star \ddagger}$ Michael S. O’Reilly, ${ }^{\ddagger}$ Blair Marshall, ${ }^{\ddagger}$ Evelyn Flynn, ${ }^{\ddagger}$ Richard-Weidong Ji, ${ }^{\ddagger}$ and Judah Folkman ${ }^{\ddagger}$ \\ *Laboratory of Angiogenesis Research, Microbiology and Tumor Biology Center, Karolinska Institute, S-171 77, Stockholm, Sweden; and \\ ${ }^{\ddagger}$ Departments of Surgery and Cell Biology, Harvard Medical School, and Children’s Hospital, Boston, Massachusetts 02115
}

\begin{abstract}
Tumor growth and metastasis are angiogenesis dependent. Previously, we reported that angiostatin, a potent angiogenesis inhibitor, produced by a primary Lewis lung carcinoma suppressed its growth of lung metastases (O'Reilly, M.S., L. Holmgren, Y. Shing, C. Chen, R.A. Rosenthal, M. Moses, W.S. Lane, Y. Cao, E.H. Sage, and J. Folkman. 1994. Cell. 79:315-328). Now we show that a shift of balance of tumor angiogenesis by gene transfer of a cDNA coding for mouse angiostatin into murine T241 fibrosarcoma cells suppresses primary and metastatic tumor growth in vivo. Implantation of stable clones expressing mouse angiostatin in C57B16/J mice inhibits primary tumor growth by an average of $77 \%$. After removal of primary tumors, the pulmonary micrometastases in $\sim 70 \%$ of mice remain in a microscopic dormant and avascular state for the duration of the experiments, e.g., 2-5 mo. The tumor cells in the dormant micrometastases exhibit a high rate of apoptosis balanced by a high proliferation rate. Our study, to our knowledge, for the first time shows the diminished growth of lung metastases after removal of the primary tumor, suggesting that metastases are self-inhibitory by halting angiogenesis. Our data may also provide a novel approach for cancer therapy by antiangiogenic gene therapy with a specific angiogenesis inhibitor. $(J$. Clin. Invest. 1998. 101:1055-1063.) Key words: angiostatin • tumor dormancy $\bullet$ angiogenesis $\bullet$ gene therapy $\bullet$ tumor metastasis
\end{abstract}

\section{Introduction}

Angiogenesis is required for a variety of physiological processes such as reproduction, development, wound repair, and tissue regeneration (1). Abnormal neovascularization can lead to progression of many diseases such as diabetic retinopathy and tumor growth (2-4). Direct experimental evidence shows that tumor growth and metastasis require new blood vessels $(1,4,5)$. An avascular tumor rarely grows to a size larger than 2-3 $\mathrm{mm}^{3}$ and contains up to a few million cells $(4,6)$. Once a tumor becomes vascularized, the expansion of tumor mass is

Address correspondence to Yihai Cao, M.D., Ph.D., The Laboratory of Angiogenesis Research, Microbiology and Tumor Biology Center, Karolinska Institute, S-171 77 Stockholm, Sweden. Phone: 46-8-7287596; FAX: 46-8-31-94-70; E-mail: yihai.cao@mtc.ki.se

Received for publication 19 August 1997 and accepted in revised form 18 December 1997.

J. Clin. Invest.

(C) The American Society for Clinical Investigation, Inc. 0021-9738/98/03/1055/09 \$2.00

Volume 101, Number 5, March 1998, 1055-1063

http://www.jci.org rapid $(1,4,6)$. The switch to the angiogenic phenotype requires both upregulation of angiogenic stimulators and in certain tumors downregulation of angiogenesis inhibitors $(1,4,6)$. Among angiogenic factors, vascular endothelial growth factor

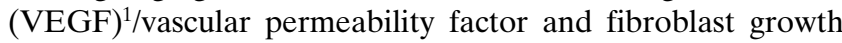
factors (FGFs) are the angiogenic factors that are most commonly expressed in tumors (7-9). Vascularized tumors may express one or more of these angiogenic factors which can function synergistically in promoting tumor growth. Although overproduction of angiogenic stimulators is necessary for the angiogenic switch, they are not sufficient for a tumor to become angiogenic. Expression of endogenous angiogenesis inhibitors must be downregulated (1, 4-6, 10-12).

In our recent reports, angiogenesis of lung metastases of a murine Lewis lung carcinoma was inhibited by angiostatin, a circulating angiogenesis inhibitor, which is a $38-\mathrm{kD}$ internal fragment of mouse plasminogen $(4,5,13)$. This fragment contains the first four disulfide-linked structures of plasminogen, known as kringle structures. In vitro, it specifically inhibits endothelial cell growth, but not proliferation of other cell types including tumor cells. Smaller fragments of angiostatin have been demonstrated to display differential effects on suppression of endothelial cell growth (14). In vivo, angiostatin suppresses neovascularization in the chick chorioallantoic membrane assay and in the mouse corneal assay (5). It seems that tumor cells do not express angiostatin molecules per se. However, they produce protease(s) which subsequently cleaves circulating plasminogen to generate angiostatin (15). It appears that protease(s) released by tumor cells is not the only source for generation of angiostatin. Metalloelastase produced by tumor-infiltrating macrophages has been found to be responsible for angiostatin production by a murine Lewis lung carcinoma (16). Thus, different types of tumors may use different strategies for generation of angiostatin. In addition to angiostatin, tumor cells also generate other angiogenesis inhibitors such as endostatin, a COOH-terminal fragment of collagen XVIII (17).

To further characterize the function of angiostatin and to examine its potential role in gene therapy of cancer, we transfected a cDNA coding for mouse angiostatin into rapidly growing murine fibrosarcoma cells and analyzed the antiangiogenic and antitumor activities in vivo.

\section{Methods}

Construction and transfection of a cDNA coding for mouse angiostatin. A cDNA coding for the mouse angiostatin was amplified by PCR using the mouse plasminogen cDNA as a template and two oligonucleotide primers (5'-ACGAAGCTTGGATCCATGGACCATAAGGAAGTA-3' [5'mAST] and 5'-ACGTCTAGAGGATCCTTAGAGGCTAGCGTAATCCGGAACATCGTATGGGTATGTG-

1. Abbreviations used in this paper: FGF, fibroblast growth factor; HA, hemagglutinin; VEGF, vascular endothelial growth factor. 
GGCAATTCCCACAACA-3' [3'mAST]), corresponding to the amino acid residues 1-6 and 461-466 of mouse plasminogen, respectively. A standard PCR protocol was used (18) and the PCR-amplified cDNA fragment was cloned into an expression vector, $\mathrm{pRC} /$ CMV (Invitrogen Corp., Carlsbad, CA). The resultant recombinant plasmid was used to transfect a murine T241 fibrosarcoma cell line by a lipofectin method as described previously (19). 4 wk after transfection, G-418 $(1 \mathrm{mg} / \mathrm{ml})$ resistant clones were selected and stained by indirect immunofluorescence with a monoclonal antibody $12 \mathrm{CA} 5 \mathrm{di}$ rected against the hemagglutinin (HA) tag as described (20). Total RNA was prepared from subconfluent cells growing in $10 \times 35 \mathrm{~mm}$ dishes according to the standard method as recommended by the manufacturer (Tel-Test, Inc.). $2 \mu \mathrm{g}$ of total RNA was applied to synthesize cDNA using the 1st-strand ${ }^{\mathrm{TM}}$ cDNA synthesis kit (Clontech, Palo Alto, CA) in a 20- $\mu$ l reaction. $1 \mu \mathrm{l}$ of cDNA was used for the PCR amplification using of $5^{\prime} \mathrm{mAST}$ and $3^{\prime} \mathrm{mAST}$. For quantitation of PCR products, amplification of actin was used as an internal control. The PCR was conducted by incubating samples for 25 cycles at $94^{\circ} \mathrm{C}$ for $1 \mathrm{~min}, 55^{\circ} \mathrm{C}$ for $1 \mathrm{~min}$, and $72^{\circ} \mathrm{C}$ for $2 \mathrm{~min}$. The amounts of actin amplified by actin specific primers were identical in each sample. This is a highly reproducible and sensitive method. We have repeated the experiments three times. The PCR-amplified DNA fragment was separated on an agarose gel and blotted with the ${ }^{32} \mathrm{P}$-labeled mouse angiostatin probe.

Western immunoblotting. Angiostatin-, vector-, and mock-transfected confluent cells were incubated for $5 \mathrm{~d}$. The conditioned media were harvested from each individual clones and $5 \mathrm{ml}$ of each conditioned medium was incubated overnight at $4^{\circ} \mathrm{C}$ with $300 \mu$ of $50 \%$ lysine-Sepharose in $50 \mathrm{mM}$ Tris- $\mathrm{HCl}, \mathrm{pH}$ 8.0. The Sepharose beads were washed three times with $50 \mathrm{mM}$ Tris- $\mathrm{HCl}, \mathrm{pH} 8.0$. The bound materials were released by addition of $50 \mu \mathrm{l}$ of SDS sample buffer followed by heating samples at $95^{\circ} \mathrm{C}$ for $3 \mathrm{~min}$. The samples were separated on a $12 \%$ SDS polyacrylamide gel and transferred onto nitrocellulose membrane. The membrane was soaked for $30 \mathrm{~min}$ at room temperature in a blocking buffer $(1 \%$ Tween $20,5 \%$ skim milk, $150 \mathrm{mM}$ $\mathrm{NaCl}$, and $20 \mathrm{nM}$ Tris-HCl, $\mathrm{pH} 7.5$ ), and was then incubated with rabbit antibodies against the HA tag (Berkeley Antibody Co., Berkeley, CA). The incubation with second antibody, alkaline phosphataseconjugated goat anti-rabbit $\mathrm{IgG}$, and development of the phosphatase reaction were carried out as described previously (19).

Animal studies. $10^{6}$ tumor cells from each transfected clone were subcutaneously implanted into 6-8-wk-old male C57B16/J mice $(n=$ 15) (The Jackson Laboratories, Bar Harbor, ME). Primary tumors were measured using digital calipers on days indicated. Animals were anesthetized with methoxyflurane (Pitman-Moore Inc., Mundelein, IL) before all procedures and observed until fully recovered. Animal studies were reviewed and approved by the animal care and use committee of Children's Hospital and are in accordance with the guidelines of the Department of Health and Human Services. Tumor sections were embedded in paraffin according to standard histologic procedures. The sections were processed and stained with a rabbit antiserum against vWf (Dako, Carpinteria, CA) as described $(13,21)$.

Cell proliferation assay. Tumor cell proliferation assays were performed as described previously (21). $10^{4}$ tumor cells of each selected clone were seeded with $10 \%$ FCS-DME onto 24 -well culture plates in triplicates. Cells were dispersed in trypsin, resuspended in PBS, and counted every 24 h by a Coulter counter (Coulter Corp., Miami, FL). For collection of conditioned media, monolayers of tumor cells in 25$\mathrm{cm}^{2}$ culture dishes were grown to confluency with $2 \mathrm{ml}$ DME medium containing $10 \%$ FCS. The medium was replaced with $2 \mathrm{ml}$ DME medium containing $5 \%$ FCS and cells were continuously incubated at $37^{\circ} \mathrm{C}$ for $72 \mathrm{~h}$. After $3 \mathrm{~d}$ of incubation, the conditioned medium was harvested and mixed with fresh DME medium in a ratio of 1:1 containing final concentrations of $10 \%$ bovine calf serum and $1 \mathrm{ng} \mathrm{bFGF} /$ $\mathrm{ml}$. The mixed medium was tested in a 72 -h bovine capillary endothelial cell proliferation assay as described $(5,14,21)$. About $1 \mathrm{ml}$ of $72-\mathrm{h}$ conditioned medium was subjected to $100 \mu \mathrm{l}$ of $20 \%$ lysineSepharose (Pharmacia Biotech, Piscataway, NJ) in $50 \mathrm{mM}$ Tris- $\mathrm{HCl}$ buffer, $\mathrm{pH} 7.5$, and rotated end-over-end at $4^{\circ} \mathrm{C}$ overnight. The Sepharose beads were washed three times with $50 \mathrm{mM}$ Tris buffer. Release of angiostatin was accomplished by incubation with $200 \mu \mathrm{l}$ of $200 \mathrm{mM} \epsilon$-amino-caproic acid (Sigma Chemical Co., St. Louis, MO) at room temperature for $20 \mathrm{~min}$. The eluted fractions from each individual clone were tested for ability to inhibit endothelial cell proliferation.

Mouse corneal micropocket assay. 6-wk-old male C57B16/J mice, each $\sim 20 \mathrm{~g}$, were subcutaneously implanted with $10^{6}$ tumor cells in $0.1 \mathrm{ml}$ PBS. After $\sim 3-5$-wk tumor cell implantation, primary tumors grew to sizes $\sim 10 \mathrm{~cm}^{3}$. A corneal micropocket was created with a cataract knife in the eye of tumor-bearing mice. Into this pocket, a sucrose aluminum sulfate (Bukh Meditec, Copenhagen, Denmark), hydron polymer pellet, type NCC (Interferon Sciences, Inc., New Brunswick, NJ) containing $80 \mathrm{ng}$ of bFGF were implanted as described previously $(5,21,22)$. The corneas of all mice were photographed by means of a slit-lamp stereoscope at a magnification of 14 .

Histology. About $10^{6}$ tumor cells were subcutaneously inoculated into 4-6-wk-old syngeneic C57Bl6/J male mice. The time necessary for a tumor containing angiostatin cDNA of clone 37 cells to reach a size $\sim 8,000 \mathrm{~mm}^{3}$ was $2-3 \mathrm{wk}$ longer than control tumors of vector and mock cells. Primary tumors were then surgically removed as described previously (5). $5 \mathrm{wk}$ after removal of primary tumors, mice were autopsied and lungs were fixed in Carnoy's fixative as described $(5,13)$. Procedures of paraffin embedding, immunohistochemical staining for apoptotic cells, and vWf have been described elsewhere $(5,13,21)$.

\section{Results}

Gene construction and expression. A PCR-amplified cDNA fragment coding for the $\mathrm{NH}_{2}$-terminal secretory signal sequence (SS), the preactivation peptide (PA), and kringle 1-4 (K1-4) regions of mouse plasminogen, and an antigenic epitope tag derived from the influenza HA, fused to the $\mathrm{COOH}$ terminus of kringle 4 , was cloned into an expression vector (pRC-CMV) under the cytomegalovirus promoter (Fig. $1 A$ ). The recombinant plasmid designated pCMV-mAST was used for transfection of a murine T241 fibrosarcoma cell line. Stable transfectants were selected by Geneticin (G-418), and the expression of recombinant mouse angiostatin was analyzed by PCR amplification (Fig. $1 B$ ) and immunofluorescence (Fig. $1, C$ and $D$ ) with an anti-HA epitope antibody. Nontransfected parental cells (Fig. 1, $B$ and $E$ ) and cells transfected with vector plasmid (Fig. 1, $B$ and $F$ ) were used as controls. An antivWf antibody was used as a control antibody which showed no staining in these tumor cells (data not shown). Immunoblotting experiments with the anti-HA polyclonal antibodies detected a 58-kD protein, the expected full-length angiostatin, in the conditioned media of the angiostatin-transfected clones (Fig. $1 \mathrm{G}$, lanes 3 and 4 ), but not in media of control clones (lanes 1 and 2). It should be emphasized that the molecular mass of recombinant mouse angiostatin was higher than that of the originally reported angiostatin fragment $(38 \mathrm{kD})$. The increased molecular mass of recombinant angiostatin could be due to the presence of the preactivation peptide and an HA tag in our construct. Both quantitative PCR and immunoblotting analyses showed that the levels of expression of angiostatin were clone $37>$ clone $31>$ clone 25 .

Suppression of primary tumor growth. The stable transfectants were subcutaneously implanted into C57B16/J syngeneic mice to assess their antiangiogenic and antitumor activities. Three independent experiments with 15 mice in each group of each independent experiment were performed in these studies. 

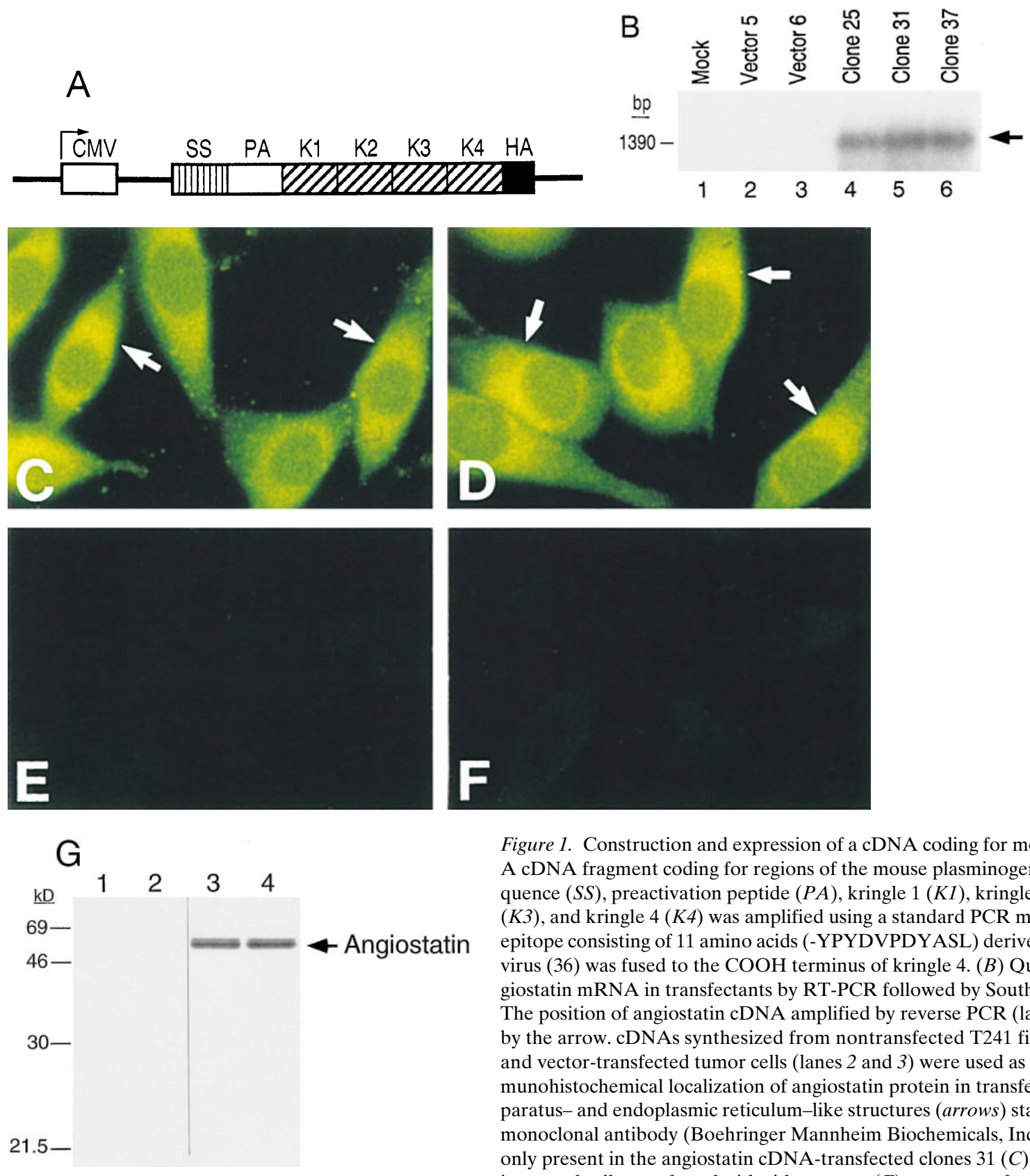

Figure 1. Construction and expression of a cDNA coding for mouse angiostatin. $(A)$ A cDNA fragment coding for regions of the mouse plasminogen including signal sequence $(S S)$, preactivation peptide $(P A)$, kringle $1(K 1)$, kringle $2(K 2)$, kringle 3 $(K 3)$, and kringle $4(K 4)$ was amplified using a standard PCR method. An HA epitope consisting of 11 amino acids (-YPYDVPDYASL) derived from the influenza virus (36) was fused to the $\mathrm{COOH}$ terminus of kringle 4. (B) Quantitation of angiostatin mRNA in transfectants by RT-PCR followed by Southern blot analysis. The position of angiostatin cDNA amplified by reverse PCR (lanes 4-6) is marked by the arrow. cDNAs synthesized from nontransfected T241 fibrosarcoma (lane 1) and vector-transfected tumor cells (lanes 2 and 3 ) were used as controls. $(C-F) \mathrm{Im}$ munohistochemical localization of angiostatin protein in transfected cells. Golgi apparatus- and endoplasmic reticulum-like structures (arrows) stained by the anti-HA monoclonal antibody (Boehringer Mannheim Biochemicals, Indianapolis, IN) were only present in the angiostatin cDNA-transfected clones $31(C)$ and $37(D)$, but not in control cells transfected with either vector $(E)$ or nontransfected parental cells $(F)$. $(G)$ Detection of angiostatin by Western immunoblot. Equal amounts of conditioned media derived from the same number of cells of clones 31 (lane 3), 37 (lane 4), mock (lane 1), and vector 5 (lane 2) were applied to lysine-Sepharose binding followed by detection of protein on SDS gel with the anti-HA antibody (see Methods). The arrow points to the position of angiostatin. Molecular mass standards are indicated on the left.

Significant inhibition of primary tumor growth $(77 \%$ inhibition versus controls) was observed in three angiostatin-expressing clones (clones 25, 31, and 37) 3 wk after tumor cell implantation (Fig. 2, $a$ and $b$ ). In contrast, primary tumors grew rapidly to volumes $>8,000 \mathrm{~mm}^{3}$ in control animals implanted with either nontransfected parental cells or with two stable clones transfected with the vector DNA (vectors 5 and 6) (Fig. $2 b$ ). Suppression of primary tumor growth paralleled expression levels of angiostatin mRNA in the transfected clones as detected by a quantitative PCR method (Fig. $1 B$ ).

Tumors derived from angiostatin-transfected clones ap- peared to be pale and flat, typical features of tumors with diminished neovascularization (see angiostatin clone 37 in Fig. 2a). In contrast, large vascularized tumors with central microhemorrhages were observed in the control groups (vector 6) (Fig. 2 a). Immunohistochemical staining with an antibody against vWf (a specific marker for endothelial cells) (23) revealed a decreased microvessel density in tumor tissues obtained from the angiostatin-transfected clones (Fig. 2, $e$ and $f$ ) as compared with control tumor tissues of mock (Fig. 2, $c$ and $f$ ) and vector plasmid-transfected clones (Fig. $2, d$ and $f)(P<0.005)$. Fig. $2 f$ represents an average of vessel density of 10 random fields in 


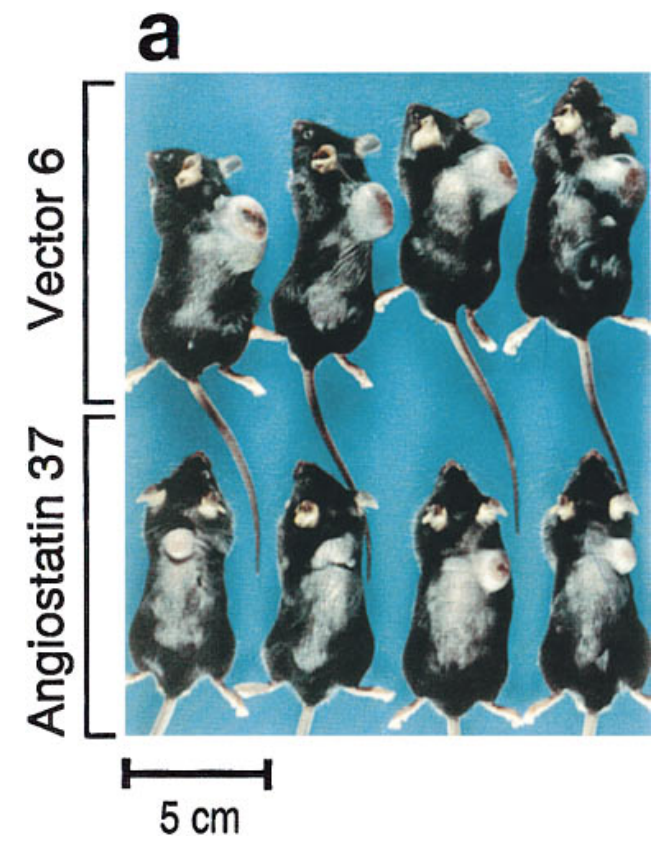

Mock

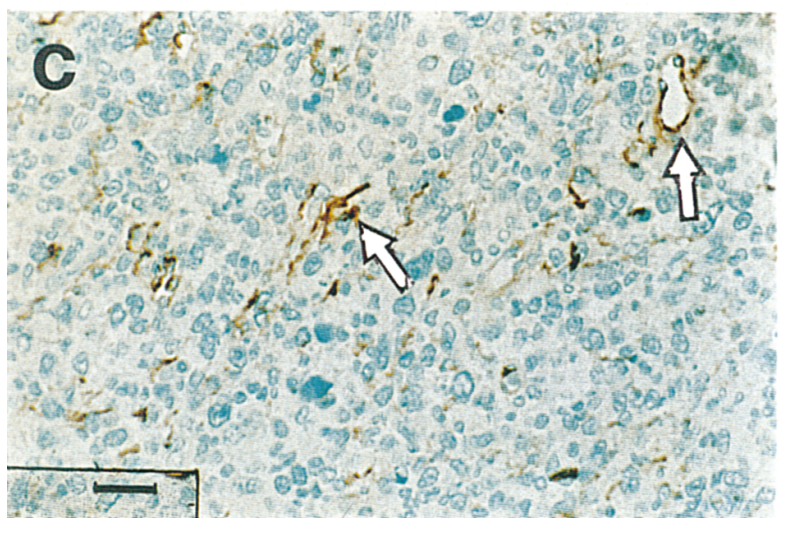

Angiostatin 37

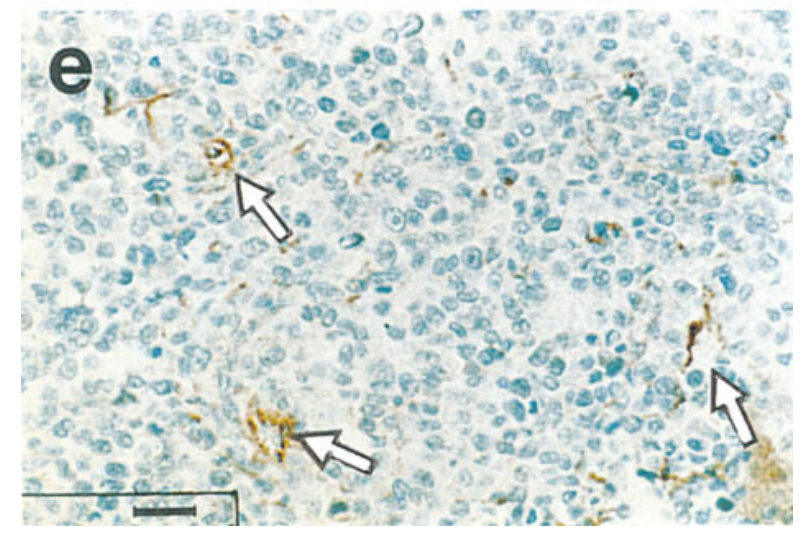

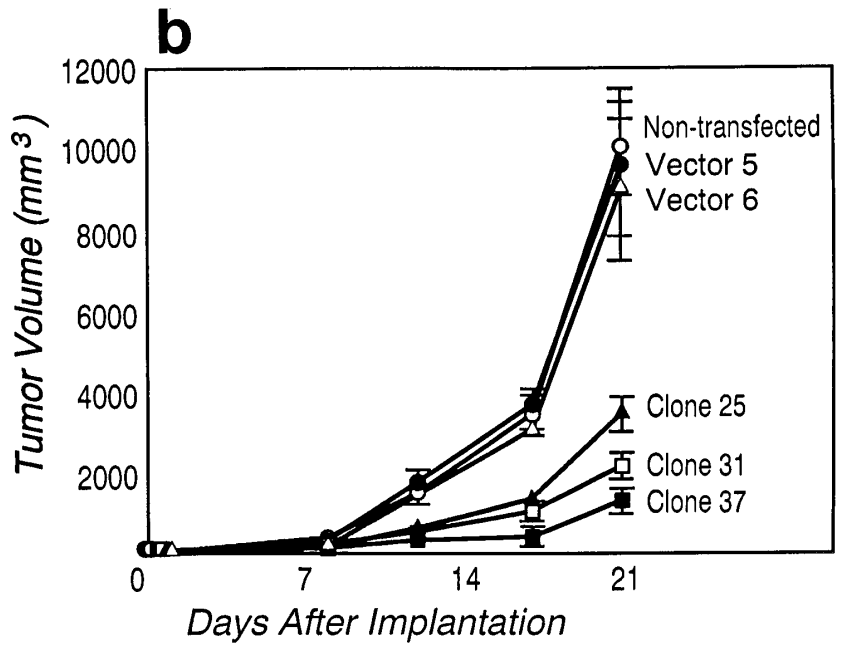

Vector 6

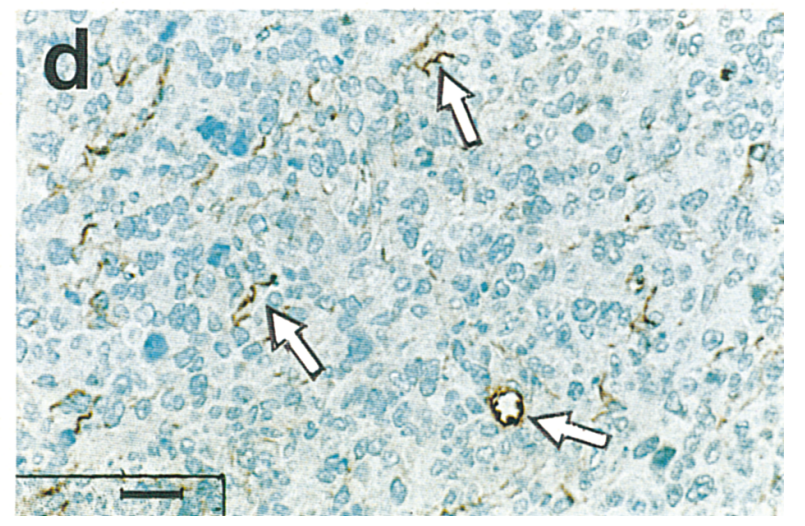

Figure 2. Suppression of primary tumor growth and tumor-induced neovascularization by angiostatin transfectants. (a) Mice bearing primary T241 fibrosarcomas. $3 \mathrm{wk}$ after implantation, subcutaneous primary tumors of vector 6 and angiostatin-transfected clone 37 growing on the backs of C57B16/J syngeneic mice were photographed. (b) Mean values ( \pm SEM) of tumor volumes $(n=15)$. Tumor volumes of angiostatin-transfected clones 25 (filled triangles), 31 (open boxes), and 37 (filled boxes) versus vector-transfected clone 5 (filled circles), clone 6 (open triangles), and untrans-

fected T421 (open circles) were determined at various time points using the formula: width ${ }^{2} \times$ length $\times 0.52$ (1). After 5 wk, mice implanted with vector 5 or mock-transfected cells formed large primary tumors with volumes $\sim 10,000 \mathrm{~mm}^{3}$. (c-e) Immunohistochemical analysis of vascularization of primary tumors by staining with the polyclonal antibody against vWf. Primary tumors were resected after 4 wk of implantation of angiostatin-transfected clone $37(e)$, vector-transfected clone $6(d)$, and mock-transfected tumor cells $(c)$, and histological sections were stained by the anti-vWf antibody. Neovascularization (arrows) of tumor tissues was revealed by the antibody (brown stain). Bar $=25 \mu \mathrm{m}$. $(f)$ Mean values $( \pm$ SEM) of microvessel density per high power field $(\times 40)$ of angiostatin-transfected clone 37 , vector 6 , and mock. Microvessels stained by the anti-vWf antibody were randomly counted from 10 different fields. $P<0.005$. 
a
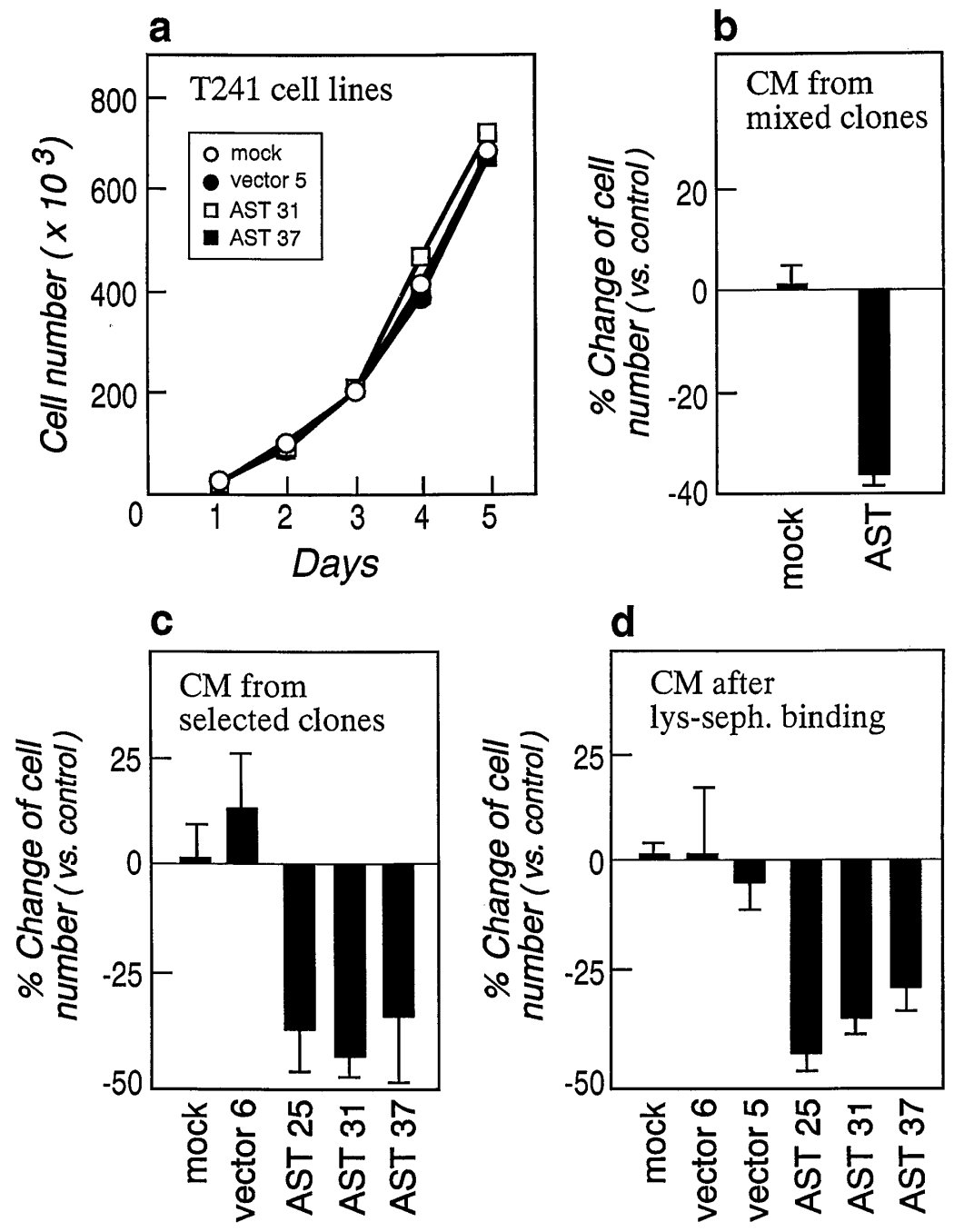

f mock

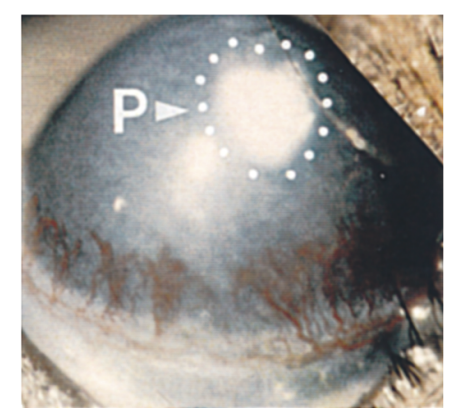

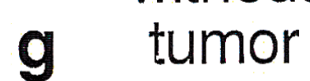

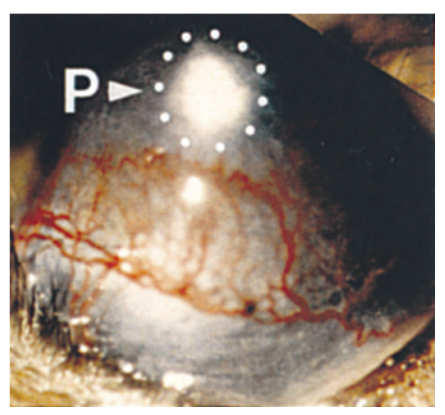

Figure 3. Proliferation assay and mouse corneal micropocket assay. (a) Growth rate of stable tumor cell lines of clone 31 (open boxes) and clone 37 (filled boxes) carrying the murine angiostatin cDNA and control cells transfected with vector (filled circles) and mock T241 (open circles) plasmids. ( $b$ and $c$ ) Inhibition of bovine capillary endothelial cell proliferation by conditioned media derived from mixed clones (b) and selected stable clones $(c)$ transfected with the CMV-mAST plasmid. Conditioned media of the vector-transfected $(c)$ and nontransfected ( $b$ and $c$ ) cells were used as controls. $(d)$ Inhibition of bovine capillary endothelial cell proliferation by conditioned media eluted from a lysineSepharose column. (e) Systemic inhibition of corneal neovascularization by angiostatin-transfected tumor of clone 37. $(f$ and $g$ ) Corneas of control C57B16/J mice bearing nontransfected parental T241 fibrosarcomas $(f)$, or without tumors $(g)$. Dots encircle the area of implanted pellet. $P$, Pellet. tumors from three mice of each group. A significant difference of vessel density was also observed between clone 37 and control tumors when compared at a similar size. For example, when control tumors were histologically compared with clone 37 at the volume of $4 \mathrm{~cm}^{3}$, the vessel density of clone 37 tumors was $\sim 50 \%$ reduced versus that of control tumors. The vessel density was quantified using three mice in each type of tumor group. Quantitation of vessel density was also performed with angiostatin-expressing clone 31 and a similar low vessel density in clone 31 tumor was found as in clone 37.

We have observed a significant suppression of tumor growth by angiostatin expressors in both immunodeficient mice and newborns of C57B16/J mice (our unpublished data). These data suggest that angiostatin expressed from transfected tumor cells inhibits tumor-induced neovascularization in vivo. We should emphasize that $50 \%$ reduction of blood vessel for- 
mation is not directly correlated to $77 \%$ reduction of tumor volume. This could be due to different growth rates between tumor cells and endothelial cells.

Inhibition of capillary endothelial cell proliferation. To further investigate whether suppression of primary tumor growth in the angiostatin-transfected clones was due to an antiangiogenic effect as opposed to direct antitumor activity, conditioned media were tested for their ability to inhibit proliferation of T241 tumor and bovine capillary endothelial cells in vitro. Although clones 31 and 37 expressed angiostatin at high levels, these cells proliferated at a similar rate as mock- and vector-transfected tumor cells (Fig. $3 a$ ). In contrast, proliferation of bovine capillary endothelial cells stimulated by $1 \mathrm{ng} / \mathrm{ml}$ bFGF was significantly inhibited by the conditioned media of angiostatin-transfected tumor cell clones (Fig. 3, $b$ and $c$, AST 25,31 , and 37 ). The endothelial inhibitory activity could be recovered after binding to lysine-Sepharose (Fig. $3 d$ ) (24). No endothelial cell inhibitory activity was found in the conditioned media of mock- and vector-transfected cells. These data are consistent with our previous results that angiostatin specifically inhibited proliferation of endothelial cells, but not tumor cells. Thus, suppression of primary tumor growth of angiostatin-transfected clones in vivo was most likely due to inhibition of tumor-induced angiogenesis by angiostatin, and not due to inhibition of tumor cell proliferation per se.

Inhibition of mouse corneal neovascularization. To demonstrate that tumor cells expressing murine angiostatin cDNA can directly inhibit angiogenesis in vivo, a sustained-release pellet containing $80 \mathrm{ng}$ of bFGF was implanted into a corneal micropocket of C57B16/J mice. At day 5 of implantation, the growth of new capillary vessels from the corneal limbus, across the cornea toward the bFGF pellet in normal mice $(n=3)$ was detected (Fig. $3 \mathrm{~g}$ ). Similarly, corneal neovascularization was also present in mice $(n=3)$ bearing nontransfected parental tumors of at least $10 \mathrm{~cm}^{3}$ (Fig. $3 \mathrm{f}$ ). Of note, the corneal vessel length of tumor-bearing animals (Fig. $3 f$ ) was shorter than that of non-tumor-bearing mice (Fig. $3 \mathrm{~g}$ ). Our preliminary findings suggest that parental T241 fibrosarcoma cells, similar to a murine Lewis lung carcinoma (5), produce an endogenous angiogenesis inhibitor which partially suppresses bFGF-induced new vessel growth in the cornea (our unpublished observation). However, corneal neovascularization was virtually absent in mice bearing angiostatin-transfected clone 37 tumors $(n=3)$ of similar sizes (Fig. $3 e$ ). Thus, angiostatin produced from the transfected tumor cells inhibited angiogenesis in vivo.

Suppression of metastatic tumor growth and tumor dormancy. To study if angiostatin produced by tumor cells affected metastatic tumor growth, we examined the lung metastases of mice after removal of primary tumors. All primary tumor resection experiments were done when tumors in each group reached a volume of $\sim 8 \mathrm{~cm}^{3}$. Three independent tumor removal experiments were performed and approximately four mice with each tumor type were used in each independent experiment. In each case, angiostatin-transfected tumors were resected $\sim 2-3$ wk later than control tumors (mock- and vector-transfected) due to their slow growth rates. Removal of parental (Fig. $4 a$, Mock) and the vector-transfected (Fig. $4 a$, Vector 5) primary T241 fibrosarcoma tumors from C57B16/J mice resulted in the exponential growth of their lung metastases. Mice died within 5 wk in these two control groups after removal of primary tumors due to their burden of lung metastases (data not shown). In contrast, lung metastases of angiostatin-transfected cells remained at microscopic sizes $(<200 \mu \mathrm{m})$ in $\sim 70 \%$ of mice and often formed perivascular cuffs of $<10$ cell layers around preexisting lung vessels (Fig. 4, $b$ and $d$ ). Dormant micrometastases of clone 37 were histologically detectable for as long as 4-5 mo after resection of primary tumors (data not shown). Whereas the lung micrometastases of clone 37 lacked new vessels, rapidly growing metastases of mock- (Fig. $4 f$ ) and vector-transfected (Fig. $4 e$ ) clones were hypervascularized with a high density of new capillary vessels as detected by immunohistochemical staining with the anti-vWf antibody.

These studies were consistent with avascular dormant micrometastases present in $\sim 70 \%$ of mice with clones 31 and 37 , but not in control groups (Table I). Similarly, significant inhibition of the growth of lung metastases was also detected in clone 25 (data not shown). Of interest, the proliferative index of the tumor cells in dormant micrometastases of clone 37 , as estimated by percentage of proliferating cell nuclear antigen positive cells $(25)$, was $\sim 35 \%$, which was virtually indistinguishable from that of the rapidly growing vascular metastases of mock- and vector 5-transfected cells (Fig. $4 \mathrm{c}$ ). The high proliferation index of dormant metastases without increasing tumor size suggested a net balance between tumor cell proliferation and death. In situ histochemical examination of apoptotic tumor cells by labeling nuclear DNA fragmentation (26) revealed that the apoptotic index of avascular metastases of clone 37 was $5.5 \pm 0.4 \%$ in contrast to $2.0 \pm 0.4$ and $2.1 \pm 0.6 \%$ in rapidly growing metastases of vector 5 - and mock-transfected cells, respectively (Fig. $4 c$ ). This is a significant difference in the rate of apoptosis. For example, an apoptotic index of $3 \%$ in developing liver $(27,28)$ has been reported to result in the loss of $25 \%$ of total hepatocytes per day. Thus, a 2.5 -fold increase of apoptotic index in dormant metastases of clone 37 could account for the differences between slow growing versus rapidly growing tumors. Similar dormant avascular micrometastases were also found to be present in lungs of mice after removal of clone 31 primary tumors.

\section{Discussion}

Spontaneously arising human and animal tumors are not often vascularized at the beginning of their development $(1,4,6)$. These in situ avascular microcancers may exist for months or years without further expansion of tumor mass (for review see reference 4). In the prevascular stage, inhibition of tumor angiogenesis from host tissues or tumor cells themselves may be prominent, although genetically altered tumor cells produce high levels of angiogenesis stimulators $(5,29-31)$. Once the expression of angiogenesis inhibitors is downregulated, dormant tumors become vascularized and the growth of tumor mass is rapid $(5,13)$. Therefore, the endothelial cell compartment acts as a "gatekeeper" in controlling tumor growth.

In our previous studies, we reported that the growth of metastases was only suppressed in mice by a primary tumor and we were unable to produce long-term dormancy of metastases with exogenous angiostatin $(5,32)$. One of the common patterns of cancer metastases is that when the primary tumor is removed (or treated with other therapy), metastases do not appear for several years (5-10 yr) (4). The cellular or molecular mechanisms of long-term dormancy of metastases in the absence of the primary tumor are unclear. Our present study provides new experimental evidence that downregulation of 


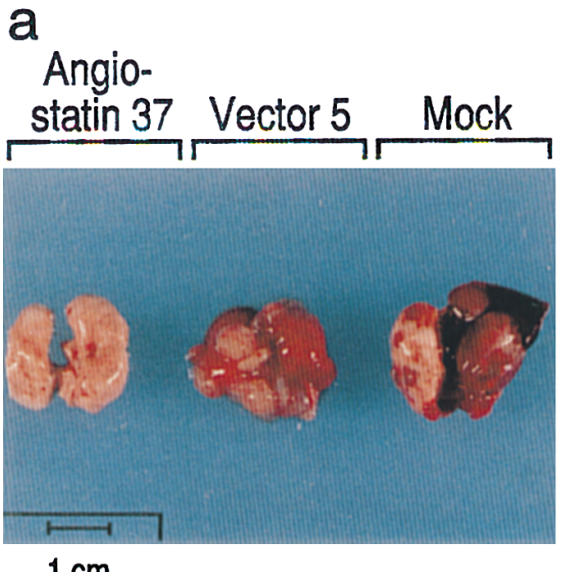

$1 \mathrm{~cm}$ b

\section{$H \& E$}

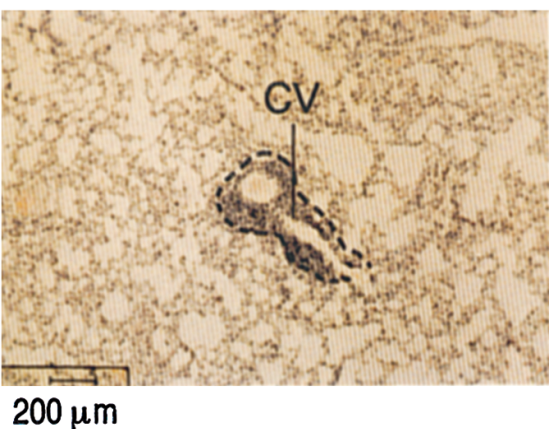

Angiostatin $37 \square$ Vector $5 \square$ Mock

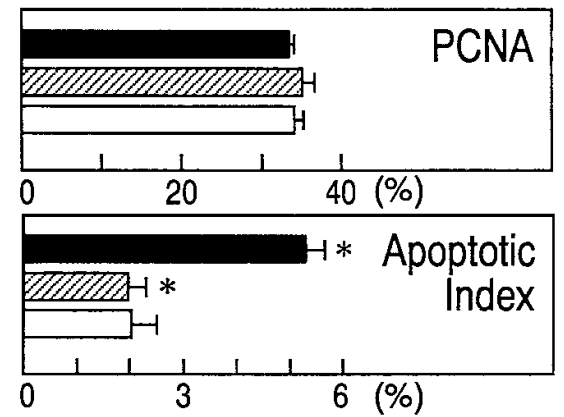

Mock
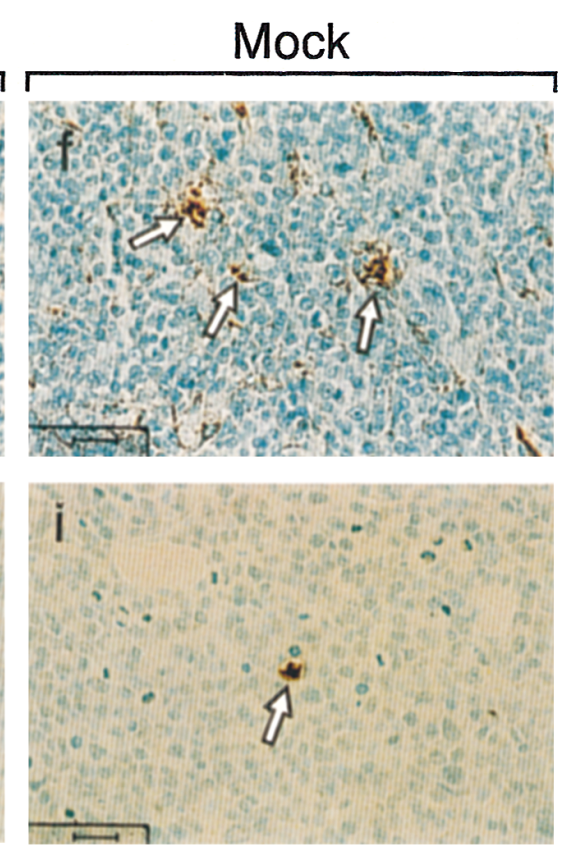
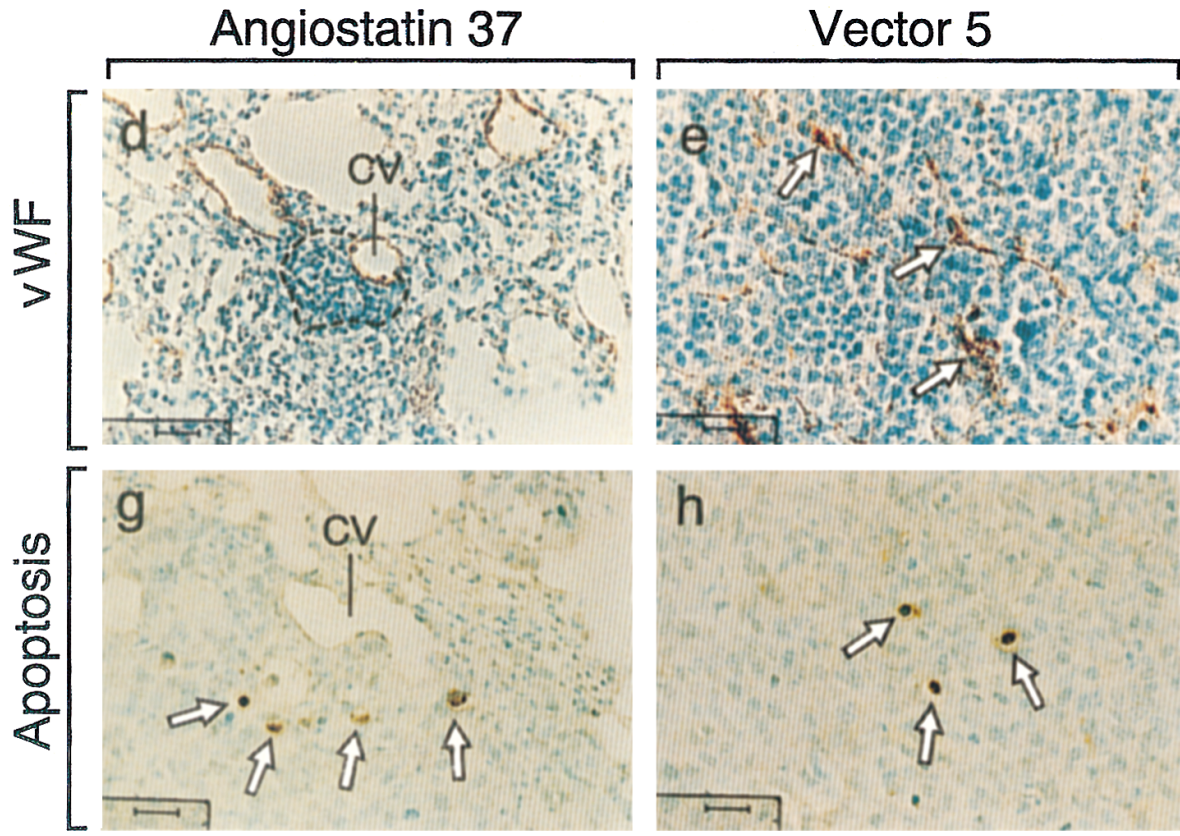

Figure 4. Histological analysis of neovascularization, cell proliferation, and apoptosis of lung metastases. (a) Lungs from mice 5 wk after removal of primary tumors of angiostatin clone 37, vector 5, and mock. Hypervascularized large metastases were visible in both lung of vector 5 and mock. Lungs of clone 37 appear to be normal. Scale bar $=1 \mathrm{~cm}$. (b) Hematoxylin and eosin staining of lung sections revealed the presence of micrometastases in mice $5 \mathrm{wk}$ after removal of the primary tumor of angiostatin clone 37. $C V$, Central vein. Dashed lines encircle the dormant micrometastasis around the preexisting vein. Scale bar $=200 \mu \mathrm{m}$. (c) Immunohistochemical analysis of proliferation and apoptotic indexes of dormant versus growing metastases. Proliferating cell nuclear antigen (PCNA) staining revealed that micrometastases of clone 37 had a similar proliferation index (35\%) as control growing metastases of vector 5 and mock. At least a 2.5 -fold increase of apoptotic tumor cell populations was observed in the dormant metastases of clone 37 as detected by TdT immunostaining. Mean \pm SEM. $n=4 . P<0.008$. $(d-f)$ Methyl green and immunohistochemical staining of lung sections with the anti-vWf antibody. Avascular micrometastases (area marked with dashed lines in $d$ ) around a central vein $(C V)$ were only present in mice of angiostatin clone $37(d)$ as compared with hypervascularized, invasive, and rapidly growing metastases of vector $5(e)$ and untransfected mock $5 \mathrm{wk}$ after removal of primary tumors $(f)$. Arrows in $e$ and $f$ indicate stained vessels in metastatic tumor tissues. ( $\mathrm{g}-i)$ Apoptotic cells (arrows) of avascular micrometastases of angiostatin clone $37(\mathrm{~g})$ and of rapidly growing metastases of vector $5(h)$ and mock $(i)$ were detected by the TdT staining technique (26). Scale bars: $b=200 \mu \mathrm{m} ; d-i=25 \mu \mathrm{m}$.

tumor angiogenesis by overexpression of angiostatin induces long-term dormancy of spontaneous metastases. The dormancy of metastases is independent of the existence of a primary tumor. These findings demonstrate that a shift of the switch of angiogenesis phenotype in metastases controls their own growth. Thus, it is not difficult to speculate that dormant metastases without a primary tumor could be due to high levels of an angiogenic inhibitor(s) production in metastatic tumor cells. In support of this notion, several human and animal tumors have been found to produce angiogenesis inhibitors (22) and their metastases may remain at the dormant state (our unpublished observation).

To inhibit tumor angiogenesis, we deliberately chose a hypervascularized murine T241 fibrosarcoma which is one of the most rapidly growing tumors in syngeneic $\mathrm{C} 57 \mathrm{~B} 16 / \mathrm{J}$ mice. These tumor cells express VEGF at high levels although they may also produce other angiogenic factors. Transfection of angiostatin cDNA in these tumor cells shifts the net balance be- 
Table I. Summary of Lung Metastases

\begin{tabular}{lccc}
\hline Cell clones & $\begin{array}{c}\text { Number of } \\
\text { experiments }\end{array}$ & $\begin{array}{c}\text { Number of } \\
\text { mice* }\end{array}$ & $\begin{array}{c}\text { Mice with avascular } \\
\text { dormant metastases }^{\ddagger}\end{array}$ \\
\hline AST 37 & 3 & 12 & 9 \\
AST 31 & 3 & 10 & 7 \\
Vector 5 & 3 & 12 & 0 \\
Vector 6 & 3 & 10 & 0 \\
Mock & 3 & 12 & 0 \\
\hline
\end{tabular}

Metastases were examined $\sim 5$ wk after removal of the primary tumor. * Three to four mice were studied in each experiment. *Lung metastases were detected by histological analysis followed by staining with the anti-vWf antibody.

tween angiogenic factors and inhibitors, and thus indirectly downregulates the angiogenic activity of VEGF and other factors. In our previous reports, high doses of angiostatin were systemically administrated in order to suppress angiogenesis and tumor growth. Our present results demonstrate that the local balance of angiogenesis in the tumor environment plays a crucial role in control of tumor growth. Other studies have shown that blockage of VEGF activity by either a neutralizing antibody or a dominant-negative VEGF receptor mutant completely suppresses VEGF-dependent tumor growth in vivo (33, 34). Suppression of primary T241 fibrosarcoma growth $(77 \%)$ by expression of angiostatin in tumor cells indicates that angiostatin is a potent antitumor factor. The antitumor growth effect of angiostatin expressed in tumor cells is unlikely due to an immune response because we have also observed a significant suppression of tumor growth by angiostatin expressors in both immunodeficient mice and newborns of C57B16/J mice (our unpublished data). It would be important to correlate the levels of angiostatin released by each individual tumor type in the circulation with its antitumor effects. However, we were unable to quantify the levels of angiostatin made by tumors and in the circulation due to lack of a specific antibody for detection. To date, an anti-mouse angiostatin antibody is not available. The anti-HA tag antibody resulted in a high background when it was applied for immunohistochemical detection of angiostatin in tumor tissues. In addition, the anti-HA antibody cross-reacts with other proteins in the circulation. In a recent similar study, Weinstat-Saslow et al. (35) showed that transfection of a cDNA coding for thrombospondin-1, another angiogenesis inhibitor, into a human breast carcinoma cell line reduced tumor growth. It appeared that a relative low number of metastatic lesions were observed in mouse lungs of angiostatin-expressing tumors as compared with control lungs. However, the lung metastases in mock- and vector-transfected tumors were difficult to count because they grew to become large confluent lesions.

It should be emphasized that angiostatin expressed in tumor cells did not completely arrest primary tumor growth. Failure of angiostatin to produce dormancy of the primary fibrosarcoma may be explained in the following way. First, the potency of inhibition of primary tumor growth correlates with the expression levels of angiostatin in three stable transfectants, suggesting that the levels of expression of angiostatin in these clones are not high enough to completely arrest primary tumor growth. Second, as primary tumors grow, the hypoxic environment may further increase the expression level of
VEGF which would oppose the inhibitory effect of angiostatin. Third, the presence of dormant metastases of angiostatin-transfected tumor cells suggests that the expression of angiostatin is greater than that of VEGF in smaller tumor populations, which produce a low concentration of angiogenic factors.

Metastatic tumor growth depends upon neovascularization in at least two steps. First, malignant cells must exit from a primary tumor into the blood circulation after the tumor becomes neovascularized. Secondly, after arrival at distant organs, metastatic cells must again induce angiogenesis for a tumor to expand to a detectable size. The long-term dormancy of lung micrometastases of the angiostatin-transfected fibrosarcoma indicates that cells can still exit from primary tumors into the circulation. It should be emphasized that the number of metastases in the lungs of mice bearing angiostatin-transfected tumors is significantly decreased compared with control mice with parental nontransfected and vector-transfected fibrosarcomas (data not shown). This finding is consistent with the immunohistological studies which revealed that angiostatintransfected primary tumors are less vascularized.

The angiostatin-induced long-term dormancy of lung metastases is equivalent to $14-15$ human years (when 1 mouse day is equivalent to $\sim 35$ human days). Occasionally, a few metastases in $\sim 30 \%$ of mice escaped from their dormancy and regrew. Although the mechanisms for regrowth of these metastases are not known, the regrowth of metastases may represent heterogeneous tumor cell populations that are selected during the process of metastases. Alternatively, regrowth of metastases may be due to the downregulation of angiostatin expression in these tumor cells. In agreement with our previous report (13), tumor cells in dormant metastases lack new vessels, but replicate as rapidly as those cells in expanding and vascularized tumors. However, in dormant metastases, proliferation reaches equilibrium with a high rate of apoptosis. Although the mechanisms of induction of tumor cell apoptosis in the dormant tumors are unclear, inhibition of neovascularization by angiostatin may restrict the supply of tumor cell survival factors provided either by the endothelial cells or by the circulation.

This study demonstrates gene transfer of a specific angiogenesis inhibitor that exclusively targets the endothelial compartment of tumor tissues. As a result, most metastases of angiostatin-transfected tumor cells are held in a harmless dormant stage at microscopic sizes. Our data suggest a novel strategy for gene therapy of cancer. Thus, combinations of conventional cytotoxic therapy with antiangiogenic gene therapy may improve efficacy of anticancer therapy. We further speculate that antiangiogenic gene therapy could be used to synergize immunotherapy.

\section{Acknowledgments}

Y. Cao is supported by the Swedish Medical Research Council, grant K97-12X-12185-01A, and the Swedish Cancer Foundation, grant 3811-B96-01XBA. This work was supported by National Institutes of Health grant RO1-CA64481 and by a grant to Children's Hospital from EntreMed Inc. (Rockville, MD).

\section{References}

1. Hanahan, D., and J. Folkman. 1996. Patterns and emerging mechanisms of the angiogenic switch during tumorigenesis. Cell. 86:353-364. 
2. Folkman, J. 1971. Tumor angiogenesis: therapeutic implications. N. Engl. J. Med. 285:1182-1186.

3. Folkman, J. 1992. The role of angiogenesis in tumor growth. Semin. Cancer Biol. 3:65-71.

4. Folkman, J. 1995. Angiogenesis in cancer, vascular, rheumatoid and other disease. Nat. Med. 1:27-31.

5. O'Reilly, M.S., L. Holmgren, Y. Shing, C. Chen, R.A. Rosenthal, M. Moses, W.S. Lane, Y. Cao, E.H. Sage, and J. Folkman. 1994. Angiostatin: a novel angiogenesis inhibitor that mediates the suppression of metastases by a Lewis lung carcinoma. Cell. 79:315-328.

6. Folkman, J. 1995. Clinical applications of research on angiogenesis. $N$. Engl. J. Med. 333:1757-1763.

7. Kandel, J., E. Bossy-Wetzel, F. Radvanyi, M. Klagsbrun, J. Folkman, and D. Hanahan. 1991. Neovascularization is associated with a switch to the export of bFGF in the multistep development of fibrosarcoma. Cell. 66:1095-1104.

8. Ferrara, N., L. Houck, L. Jakeman, and D.W. Leung. 1992. Molecular and biological properties of the vascular endothelial cell growth factor family of proteins. Endocr. Rev. 13:18-32.

9. Dvorak, H.F., L.F. Brown, M. Detmar, and A.M. Dvorak. 1995. Vascular permeability factor/vascular endothelial growth factor, microvascular hyperpermeability, and angiogenesis. Am. J. Pathol. 146:1029-1039.

10. Rastinejad, F., P. Polverini, and N.P. Bouck. 1989. Regulation of the activity of a new inhibitor of angiogenesis by a cancer suppressor gene. Cell. 56: 345-355.

11. Sharpe, R.J., H.R. Byer, C.F. Scott, S.I. Bauer, and T.E. Maione. 1990. Growth inhibition of murine melanoma and human colon carcinoma by recombinant human platelet factor 4. J. Natl. Cancer Inst. 82:848-853.

12. Brooks, P.C., A.M. Montgomery, M. Rosenfelt, T. Hu, G. Klier, and D.A. Cheresh. 1994. Integrin $\alpha_{v} \beta_{3}$ antagonists promote tumor regression by inducing apoptosis of angiogenic blood vessels. Cell. 79:1157-1164.

13. Holmgren, L., M.S. O'Reilly, and J. Folkman. 1995. Dormancy of metastases: balanced proliferation and apoptosis in the presence of angiogenesis suppression. Nat. Med. 1:149-153.

14. Cao, Y., R.-W. Ji, D. Davidson, J. Schaller, D. Marti, S. Sohndel, S.G. McCance, M.S. O'Reilly, M. Llinás, and J. Folkman. 1996. Kringle domains of human angiostatin: characterization of the anti-proliferative activity on endothelial cells. J. Biol. Chem. 271:29461-29467.

15. Gately, S., P. Twardowski, M.S. Stack, M. Patrick, L. Boggio, D.L. Cundiff, H.W. Schnaper, L. Madison, O. Volpert, N. Bouck, et al. 1996. Human prostate carcinoma cells express enzymatic activity that converts human plasminogen to the angiogenesis inhibitor angiostatin. Cancer Res. 56:4887-4890.

16. Dong, Z., R. Kumar, X. Yang, and I.J. Fidler. 1997. Macrophage-derived metalloelastase is responsible for the generation of angiostatin in Lewis lung carcinoma. Cell. 88:801-810.

17. O'Reilly, M.S., T. Boehm, Y. Shing, N. Fukai, G. Vasios, W.S. Lane, E. Flynn, J.R. Birkhead, B.R. Olsen, and J. Folkman. 1997. Endostatin: an endogenous inhibitor of angiogenesis and tumor growth. Cell. 88:277-285.

18. Cao, Y. 1990. Direct cloning of a chimera gene fused by the polymerase chain reaction. Technique (Phila.). 2:109-111.

19. Cao, Y., and R.F. Pettersson. 1993. Release and subcellular localization of acidic fibroblast growth factor expressed to high levels in HeLa cells. Growth Factors. 8:277-290.
20. Chen, Y.T., C. Holcomb, and H.P. Moore. 1993. Expression and localization of two molecular weight GTP-binding proteins, Rab8 and Rab10, by epitope tag. Proc. Natl. Acad. Sci. USA. 90:6508-6512.

21. Cao, Y., C. Chen, J.A. Weatherbee, M. Tsang, and J. Folkman. 1995. gro- $\beta$, a -C-X-C- chemokine, is an angiogenesis inhibitor that suppresses the growth of Lewis lung carcinoma in mice. J. Exp. Med. 182:2069-2077.

22. Chen, C., S. Parangi, M. Tolentino, and J. Folkman. 1995. A strategy to discover circulating angiogenesis inhibitors generated by human tumors. Cancer Res. 55:4230-4233.

23. Ginsburg, D., R.I. Handin, D.T. Bonthron, T.A. Dolon, G.A.P. Bruns, S.A. Latt, and S.H. Orkin. 1985. Human von Willebrand factor (vWF): isolation of complementary DNA (cDNA) clones and chromosomal localization. Science. 288:140-145.

24. Ponting, C.P., J.M. Marshall, and S.A. Cederholm-Williams. 1992. Plasminogen: a structure review. Blood Coagul. Fibrinolysis. 3:605-614.

25. Key, G., M.H.G. Becher, B. Beron, M. Duchrow, G. Schluter, H.-D Flad, and J. Gerdes. 1993. New Ki-67-equivalent murine monoclonal antibodies (MIB 1-3) generated against bacterially expressed parts of the Ki-67 cDNA containing three 62 base pair-repetitive elements encoding for the Ki-67. Lab. Invest. 68:629-636.

26. Gavrieli, Y., Y. Sherman, and S.A. Ben-Sasson. 1992. Identification of programmed cell death in situ via specific labeling of nuclear DNA fragmentation. J. Cell Biol. 119:493-501.

27. Bursh, W., S. Paffe, B. Putz, G. Barthel, and R. Schulte-Hermann. 1990 Determination of the length of the histological stages of apoptosis in normal liver and altered hepatic foci of rats. Carcinogenesis. 11:847-853.

28. Barres, B.A., I.K. Hart, H.S.R. Coles, J.F. Burne, J.F. Voyvodic, W.D. Richardson, and M.C. Raff. 1992. Cell death and control of cell survival in the oligodendrocyte lineage. Cell. 70:31-46.

29. Dameron, K.M., O.V. Volpert, M.A. Tainsky, and N. Bouck. 1994. Control of angiogenesis in fibroblasts by p53 regulation of thrombospondin-1. Science. 265:1582-1584.

30. Auerbach, W., and R. Auerbach. 1994. Angiogenesis inhibition: a review. Pharmacol. Ther. 63:265-311.

31. Shweiki, D., A. Itin, D. Soffer, and E. Keshet. 1992. Vascular endothelial growth factor induced by hypoxia may mediate hypoxia-induced angiogenesis. Nature. 359:843-845

32. O'Reilly, M.S., L. Holmgren, C. Chen, and J. Folkman. 1996. Angiostatin induces and sustains dormancy of human primary tumors in mice. Nat. Med. 2:689-692.

33. Kim, K.J., B. Li, J. Winer, M. Armanini, N. Gillett, H.S. Philips, and N. Ferrara. 1993. Inhibition of vascular endothelial growth factor-induced angiogenesis suppresses tumor growth in vivo. Nature. 362:841-844.

34. Millauer, B., L.K. Shawver, K.H. Plate, W. Risau, and A. Ullrich. 1994. Glioma growth inhibited in vivo by a dominant-negative Flk-1 mutant. Nature 367:576-579.

35. Weinstat-Saslow, D.L., V.S. Zabrenetzky, K. VanHoutte, W.A. Frazier, D.D. Roberts, and P.S. Steeg. 1994. Transfection of thrombospondin 1 complementary DNA into a human breast carcinoma cell line reduces primary tumor growth, metastatic potential, and angiogenesis. Cancer Res. 54:6504-6511.

36. Gu, Y., C.W. Turk, and D.O. Morgan. 1993. Inhibition of CDK2 activity in vivo by an associated $20 \mathrm{~K}$ regulatory subunit. Nature. 366:707-710. 